\title{
Novel ultrasound-responsive chitosan/pefluorohexane nanodroplets for image-guided smart delivery of an anticancer agent: curcumin
}

Fatemeh Baghbani $^{\mathrm{a}}$, Fathollah Moztarzadeh ${ }^{\mathrm{a}}$, Samaneh Hadian ${ }^{\mathrm{b}}$

${ }^{a}$ Biomaterials Group, Faculty of Biomedical Engineering (Center of Excellence), Amirkabir University of Technology, Tehran, Iran

${ }^{\mathrm{b}}$ Laboratory of Microanalysis, Institute of Biochemistry and Biophysics, University of Tehran, P.O. Box 131451384, Tehran, Iran.

Corresponding author: Tel: (+98)216454-2393

E-mail address: f.baghbani@aut.ac.ir (f.baghbani)

\begin{abstract}
Ultrasound-responsive nanodroplets are a class of new emerging smart drug delivery systems which provide image-guided nano-therapy of various diseases, especially cancers. Here, we developed multifunctional smart curcumin-loaded chitosan/perfluorohexane nanodroplets for contrast-ultrasound imaging and on-demand drug delivery. The nanodroplets were synthesized via nanoemulsion process. The optimal formulation with the size of $101.2 \mathrm{~nm}$ and $77.8 \%$ curcumin entrapment was chosen for release study and cytotoxicity evaluation. Sonication at the frequency of $1 \mathrm{MHz}, 2 \mathrm{~W} / \mathrm{cm}^{2}$ for $4 \mathrm{~min}$ triggered the release of $63.5 \%$ of curcumin from optimal formulation (Cur-NDs-2). Ultrasound aided release study indicated that the concentration of perflourohexane and the degree of acoustic droplet vaporization play important role in ultrasound-active drug release. B-mode ultrasound imaging confirmed strong ultrasound contrast of chitosan nanodroplets even at low concentrations via droplet to bubble transition. Finally, cytotoxicity of the ultrasound-responsive nanodroplets in the presence of ultrasound was evaluated in-vitro on 4T1 human breast cancer cells. Cell growth inhibitory effects of curcuminloaded nanodroplets significantly increased by ultrasound exposure. According to the obtained results, these ultrasound responsive curcumin-loaded chitosan/perfluorohexane nanodroplets have a great potential for imaged-guided cancer therapy.
\end{abstract}

Keywords: Chitosan/pefluorohexane nanodroplets; Ultrasound-responsive; Contrast agent; Curcumin; Smart delivery; Breast cancer; 


\section{Introduction}

Ultrasound is a widely available, noninvasive, and cost-effective diagnostic modality [1-4]. A novel ultrasound-mediated chemotherapeutic modality is based on systemic injection of phaseshift drug-loaded nanodroplets that vaporize and convert into microbubbles under the action of ultrasound. Acoustic phase shift nanodroplets effectively accumulate in tumor tissue by passive or active targeting and then convert into microbubbles in situ by ultrasound $[5,6]$. Under the action of ultrasound, perfluorocarbon (PFC) nanodroplets vaporize into gas bubbles via acoustic droplet vaporization or ADV which results in triggered release of encapsulated drugs from nanodroplets $[7,8]$. Expansion of nanodroplets from ADV induces mechanical tissue erosion and cell damage [9-10] and promotes vascular permeability and ultrasound ablation for tumor tissue $[11,12]$. These features of PFC nanodroplets make them promising candidates for overcoming the limitations of contrast bubbles such as very short circulation time (minutes) and large size (within micron range) which hinder their effective extravasation into tumor tissue, which is essential for effective drug targeting [5,6].

The polyphenol curcumin (1,7-bis(4-hydroxy-3-methoxyphenyl)-1,6-heptadiene-3,5-dione), is one of the most active component of the perennial herb curcuma longa (commonly known as turmeric) [13]. Curcumin has a wide range of therapeutic properties such as anticancer [14,15], antioxidant [15,16], anti inflammatory [17], anti-arthritic [18], anti-ischaemic [19], and antiamyloid [20]. Curcumin suppresses many types of cancers [21], cures multidrug-resistant cancers [22], and shows a synergistic antitumor effect with other anticancer agents. However, curcumin is a hydrophobic molecule and has a poor aqueous solubility [23] which eliminates its bioavailability and therapeutic efficiency [24]. Therefore, developing a delivery system to improve the aqueous solubility and stability and subsequently the bioavailability of curcumin is important.

Ultrasound-responsive nanodroplets comprise a perfluorocarbon (PFC) core and a stabilizing shell of lipid, polymer and/or protein. In first generation of perfluorocarbon nanodroplets, perfluoropentane (PFP) was used as a droplet core [7,25-31]. PFP has a low boiling temperature $\left(29^{\circ} \mathrm{C}\right)$; therefore, PFP droplets have low stability. They easily turn to foam in blood circulation before reaching the target tissue and it is hard to control their droplet-to-bubble transition [7,29]. In order to obtain nanodroplets with higher stability, perfluorohexane (PFH) was chosen for the droplet core in this work because of its higher boiling point $\left(58-60{ }^{\circ} \mathrm{C}\right)$ compared to other common perfluorocarbons including perfluoropentane.

Various PFC nanodroplet formulations have been developed for ultrasound controlled drug delivery and gene delivery. Most of them are comprised a block copolymer shell such as poly(ethylene oxide)-co-poly(L-lactide) (PEG-PLLA) or poly(ethylene oxidecopolycaprolactone (PEG-PCL) [26,29-31], a lipid shell (DPPC, DSPE-PEG/cholesterol) [32], a protein shell (lung surfactant, albumin) [33-35] or a surfactant shell (perfluroocatanoic acid) [36]. In order to design a cost effective nanodroplet formulation with high cucrcumin entrapment

efficiency and no in-vivo toxicity, we considered using the well-known biocompatible 
polysaccharide, chitosan as the stabilizing shell. Chitosan, [(1-4)-2-amino-2-deoxy-_-d-glucan], is a cationic linear polysaccharide with interesting physicochemical properties such as its abundance, flexibility, nontoxicity, hydrophilicity, biocompatibility [37], biodegradability [38], and high resistance to heat [39] which makes it suitable for biomedical application including drug delivery [40]. Also, chitosan binds curcumin with high affinity at considerably high $\mathrm{pH}(\mathrm{pH}$ $=7.0-10.5)$ through its functional amine groups [41]. Moreover, chitosan effectively interacts with different surfactants in aqueous solutions [42]. This could be used to tailor the size and other characteristics of nanoparticles.

The aim of the current study was to formulate and characterize a new curcumin-loaded multifunctional nanodroplet formulation composed of a chitosan/surfactant shell and a perfluorohexane $(\mathrm{PFH})$ core to improve the bioavailability of curcumin and enhance its anti-tumor efficiency by image guided ultrasound-mediated triggered drug release.

\section{Experimental}

\subsection{Materials}

LMW chitosan, curcumin, Perfluorohexane and Tween 20, lecithin, phosphate-buffered saline (PBS, pH 7.4) and dialysis membranes (molecular weight cutoff, 12000) were purchased from Sigma-Aldrich (St. Louis, MO, USA). All other chemicals and solvents were obtained commercially as analytical-grade reagents. 4T1 human breast cancer cells were obtained from Pasteur Institue (Tehran, IR). Ultrasound was generated via a 1-MHz therapeutic unit (SM3670, Shrewsbury Medical Ltd., Shropshire, UK). 12-MHz linear transducer (Acuson Sequoia 512, Siemens, Mountain View, CA) was used to monitor ADV of nanodroplets.

\subsection{Preparation of curcumin-loaded Citosan/PFH nanodroplets}

Curcumin-loaded chitosan-stabilized PFH nanodroplets were prepared via nano-emulsion process [43]. Briefly, curcumin and lecithin (surfactant) solution in $98 \%$ ethanol were added to perfluorohexane (PFH) and distilled deionized (DD) water under stirring and homogenized for $2 \mathrm{~min}$ at 24,000 rpm using Ultra-Turrax SG215 homogenizer. Then, polymer solution (chitosan $1.5 \% \mathrm{w} / \mathrm{v}$ in $1 \% \mathrm{vlv}$ acetic acid, $\mathrm{pH}, 5$ ) was added drop-wise to the emulsion while homogenized at 13,000 rpm for $3 \mathrm{~min}$. For co-surfactant inclusion, Tween 20 was first added to the mixture of PFH and DD water, then the rest of the precursors were added as before. In order to separate the free curcumin from curcumin-loaded nanodroplets, the nanodroplets were centrifuged, then they were dispersed in PBS and ethyl acetate (1:1). The mixture was shaken to dissolve the free curcumin content in ethyl acetate and finally, the ethyl acetate phase containing free curcumin was separated.

\subsection{Characterization of Citosan/PFH nanodroplet}

\subsubsection{Phsicochemical properties}


The average size, polydispersity index and zeta potential of nanodroplets were measured dynamic light scattering at a scattering angle of $90^{\circ}$ using Zeta-sizer $3000 \mathrm{HS}$ (Malvern Instruments, Malvern, UK).

Morphology and shape of curcumin-loaded nanodroplets were observed using transmission electron microscopy, TEM, (H-7650; Hitachi, Tokyo, Japan). Sample was prepared for imaging, by pouring one drop of the solution on a 400-mesh carbon-coated copper grid and drying at room temperature.

The structural features of pure curcumin, chitosan and curcumin-loaded chitosan nanodroplets were determined by Fourier transform infrared (FTIR, Bomem MB 100 spectrometer, Bruker IFS 28 ) in the $4500-400 \mathrm{~cm}^{-1}$ spectral range using $\mathrm{KBr}$ pellets.

\subsubsection{Determination of entrapment efficiency}

To determine the entrapment efficiency of curcumin, nanodroplets were freeze dried (1 mg) and dispersed in $1 \mathrm{ml}$ PBS, and then $1 \mathrm{ml}$ of ethyl acetate was added. The mixture was shaken and ethyl acetate phase being separated. Free curcumin content in ethyl acetate phase was determined using a UV-Vis spectrophotometer at $424 \mathrm{~nm}$ (U.V-1601; Shimadzu, Japan). Samples were prepared and measured in triplicate. The percentage of drug entrapment efficiency (EE) was calculated using the following equation:

Entrapment Efficiency $(\%)=\frac{(\text { Total amount of drug added })-(\text { Free amount of drug })}{\text { Total amount of drug added }} \times 100$

\subsubsection{Stability of nanodroplets}

Nanodroplets were suspended in phosphate-buffered saline (PBS) at $\mathrm{pH} 7.4$ and incubated at 4 ${ }^{\circ} \mathrm{C}$ for predetermined time intervals. The stability of nanodroplets was determined by changes in their size and drug entrapment efficiency.

\subsubsection{Stability of curcumin loaded into nanodroplets}

To estimate the stability of curcumin in its free form and loaded into nanodroplets, they were mixed with PBS ( $\mathrm{pH} \mathrm{7.2),} \mathrm{at} \mathrm{the} \mathrm{same} \mathrm{curcumin} \mathrm{concentration} \mathrm{and} \mathrm{poured} \mathrm{into} \mathrm{microcentrifuge}$ tubes. The samples were incubated at $37^{\circ} \mathrm{C}$ under shaking at $100 \mathrm{rpm}$. The concentration of remained curcumin at various time intervals was measured using a UV-vis spectrophotometer $(\lambda=424 \mathrm{~nm})$. Each sample was tested in triplicate. The percentage of remaining curcumin was calculated as followed:

Remaining curcumin $(\%)=\frac{\text { amount of curcumin at a fixed time }}{\text { Total amount of curcumin added }} \times 100$ 


\subsubsection{In-vitro drug release study 2.3.5.1. Passive Drug release}

Release study was performed using dialysis sack method by dialysis tubing (MW cutoff 12000 $\mathrm{g} / \mathrm{mol}$, Sigma, Canada). Two $\mathrm{ml}$ of nanodroplet solution was placed in dialysis membrane and immersed in $20 \mathrm{ml}$ phosphate buffer pH 7.4 and citrate buffer $\mathrm{pH} 5.4$ containing $0.1 \%$ Tween 20. The release study was carried out in a shaker incubator (MS MP8 Wise Stir Wertheim, Germany) with shaking rate of $100 \mathrm{rpm}$ at $37{ }^{\circ} \mathrm{C}$ for $24 \mathrm{~h}$. Two $\mathrm{ml}$ samples were withdrawn in predetermined time intervals and replaced with an equivalent volume of fresh buffer. The amount of released curcumin was measured at $424 \mathrm{~nm}$ using UV-vis spectrophotometer (U.V1601; Shimadzu, Japan). To ensure that sustained release profile is not due to membrane, curcumin dispersion in the same concentration with curcumin nanodroplets was studied under the same condition for release.

\subsubsection{Ultrasound-induced drug release}

To determine the influence of $1-\mathrm{MHz}$ ultrasound on curcumin release from nanodroplets, the nanodroplet solution was purred into Plexiglas scaffold with a latex cover and placed into a water bath at $37{ }^{\circ} \mathrm{C}$, and then was sonicated for $0.5,1,2,4$ and 7 min with $2 \mathrm{~W} / \mathrm{cm}^{2}$. After processing, the nanodroplet solution was withdrawn, and centrifuged at $11000 \mathrm{rpm}$, then the freecurcumin concentration was determined as described above. Each experimental condition was repeated 3 times. Continuous wave $1-\mathrm{MHz}$ ultrasound was generated by an $1-\mathrm{MHz}$ therapeutic unit (SM3670, Shrewsbury Medical Ltd., Shropshire, UK)

\subsubsection{Measurement of echogenicity}

Droplet to bubble transition of nanodroplets at $37{ }^{\circ} \mathrm{C}$ was monitored by ultrasound imaging using a $12-\mathrm{MHz}$ linear transducer (Acuson Sequoia 512, Siemens, Mountain View, CA). Mechanical index of $<0.8$ was used to induce cavitation in nanodroplets.

\subsubsection{Hemolytic activity}

To evaluate the hemolytic activity of curcumin-loaded nanodroplets human blood from healthy volunteers was collected and centrifuged at $1500 \mathrm{rpm}$ for $10 \mathrm{~min}$. The RBCs sediments were washed three times with sterile normal saline $(0.9 \% \mathrm{NaCl})$ and were resuspended with normal saline. Hemolytic activity of different concentration of free curcimin and curcumin-loaded nanodroplet $(1,2,4,7$, and $10 \mathrm{mg} / \mathrm{ml})$ in erythrocytes suspension was analyzed. Complete hemolysis for the hemolytic control was achieved by adding Triton X-100 to a same amount of erythrocytes suspension. Hemolytic activity of drug-free chitosan nanodroplets formulation (blank nanoparticles) was also evaluated. The suspensions were incubated for $90 \mathrm{~min}$ at $37^{\circ} \mathrm{C}$. The reaction was terminated using $50 \mu \mathrm{l}$ of glutaraldehyde $(2.5 \%)$. The samples were then 
centrifuged at $11000 \mathrm{rpm}$ for 30 minutes and the absorbance of the supernatant which was correlated with the concentration of released hemoglobin was measured using UV-vis spectrophotometer at a wavelength of $540 \mathrm{~nm}$. The experiment was carried out in the triplicate.

\subsubsection{Cytotoxicity}

To examine the cytotoxicity of curcumin nanodroplets, 4T1 human breast cancer cells were seeded at a density of $1 \times 10^{4}$ cells /well in 96-well plates and incubated at $37{ }^{\circ} \mathrm{C}$ in a humidified air containing $5 \% \mathrm{CO}_{2}$ for $24 \mathrm{~h}$. Then, curcumin-loaded nanodroplets or free curcumin were added to the wells at the concentrations of $0.004,0.04$, or $0.4 \mu \mathrm{g} / \mathrm{ml}$ and incubated for $24 \mathrm{~h}$ and $48 \mathrm{~h}$. Blank control wells ( $0 \%$ cytotoxicity) received $100 \mu 1$ fresh medium. The cytotoxicity in combination with ultrasound was also evaluated. The same dosages of nanodroplets in another 96-well plate were exposed to $1 \mathrm{MHz}$ ultrasound $\left(2 \mathrm{~W} / \mathrm{cm}^{2}\right)$ for $4 \mathrm{~min}$. The concentration of curcumin of each nanodroplets was the same as the free Cur. 24 and 48 hours post-incubation, 10 $\mu 1$ of $5 \mathrm{mg} / \mathrm{ml}$ MTT solution in PBS (Sigma) was added to wells, and then further incubated for $4 \mathrm{~h}$. Then, the plates were centrifuged, the supernatants were removed, and the formazan crystals were dissolved using $100 \mu \mathrm{L}$ of dimethyl sulfoxide (DMSO). The absorbance was read by a microplate reader at $570 \mathrm{~nm}$ and at a reference wavelength of $650 \mathrm{~nm}$. Each treatment was performed at 5 replicates. The schematic of ultrasound set up used to sonicate the cell plates, is shown in Fig. 1.

\subsubsection{Statistical analysis}

The data are presented as the mean value with standard deviation ( $n=3-5$, mean \pm SD), and statistical significance was determined by pair sample t-test analysis of variance. $P$ values less than 0.05 was considered to be statistically significant.

\section{Results and discussion}

\subsection{Preparation of curcumin-loaded chitosan/PFH nanodroplets}

Novel PFH nanodroplets covered by a chitosan/surfactant shell were developed for smart controlled curcumin delivery.

\subsection{Size and morphology of nanodroplets}

The average size, polydispersity index, and zeta potential of the nanodroplets, before and after drug loading, are presented in Table 1. The average diameter of blank nanodroplets was $163 \mathrm{~nm}$. After loading of curcumin into the nanodroplets, the average size of droplets increased $(255 \mathrm{~nm}$, formulation Cur-NDs-1), and it was proportional to the amount of drug loaded (data not shown).

The nanodroplets were synthesized via nanoemulsion process; critical micellar concentration of lecithin was approximately $0.9 \mathrm{mg} / \mathrm{ml}$ and stable $\mathrm{PFH}$ nanodroplets were formed at this surfactant concentration. In order to obtain smaller droplets, the formulation was improved by 
inclusion of a co-surfanant. Tween 20 was used as co-surfactant. By addition of Tween 20, the particle size decreased from $255 \mathrm{~nm}$ (no co-surfactant) to $101.2 \mathrm{~nm}$ (formulation Cur-NDs-2) but further increase of Tween 20 resulted in larger nanodroplets (184.8 nm, formulation Cur-NDs-3). By localization of this amphiphilic molecule along with lecithin in the PFH-water interface, the interfacial tension between the oil and water phase decreases which results in formation of smaller droplets. Further increase in Tween 20 concentration increases the interaction between the hydrophilic chains of particles which may result in agglomeration of nanodroplets and formation of larger particles [44].

The unloaded chitosan nanodroplets had a positive zeta potential $(+64.01)$ which considerably decreased with loading of curcumin (+28.37, formulation Cur-NDs-1), indicating the presence of electrostatic interactions between the positive amino groups of chitosan and the negative phenolic groups of curcumin. This result indicates that there is an optimal value for curcumin loading, because by further increase in the amount of loaded curcumin, the surface charge-zeta potentials- decreases, favoring the aggregation of nanodroplets and lowering the physical stability of the nanoemulsion.

The morphology of curcumin-loaded nanodroplets (formulation Cur-NDs-2) which was observed using TEM (Fig. 2a) showed a well defined core-shell structure with a shell thickness of about 6 $\mathrm{nm}$. The nanodroplets did not have a spherical shape in TEM image which could be because of the liquid-gas conversion of PFH core under high vacuum when using TEM [45].

\subsection{Structural analysis}

FTIR analysis was performed to identify the major interactions involved in formation of curcumni-chitosan nanoparticle. FTIR spectra of curcumin, chitosan and curcumin-loaded chitosan nanodroplets are presented in Fig 2b. Three characterization peaks of curcumin at 1632 $\mathrm{cm}^{-1}, 1286 \mathrm{~cm}^{-1}$ and $1157 \mathrm{~cm}^{-1}$ can be attributed to $v(\mathrm{C}=\mathrm{C})$, enol $v(\mathrm{C}-\mathrm{O})$ and $v(\mathrm{C}-\mathrm{O}-\mathrm{C})$ of the phenyl group mixed with methoxy group. The sharp peak at $3520 \mathrm{~cm}^{-1}$ can be assigned to $v(\mathrm{O}-\mathrm{H})$.

FTIR spectrum of chitosan showed characteristic peaks at 1035, 1380, 1424 and 1636 and 2921 $\mathrm{cm}^{-1}$ attributed to $v(\mathrm{C}-\mathrm{O}-\mathrm{C})$ and $v(\mathrm{NH} 2), \delta\left(\mathrm{CH}_{2} \mathrm{OH}\right)$ and $v(\mathrm{C}=\mathrm{O}-$ Amide $\mathrm{I}$ band $)$ in $\mathrm{NHCOCH}_{3}$ and $v_{\mathrm{as}}\left(\mathrm{CH}_{2} \mathrm{OH}\right)$ groups. The broad peak in the range of $3500-3000 \mathrm{~cm}^{-1}$ was correspond to the $-\mathrm{OH}$ group.

The spectrum of Cur-chitosan nanodroplets showed that most characteristic peaks of curcumin were overlapped or merged by the transmittance peaks of chitosan. The transmittance peak of curcumin at $1157 \mathrm{~cm}^{-1}$ was observed in the spectrum of nano-formulation demonstrating the presence of curcumin. In Cur-chitosan nanodroplets, the peak at $1636 \mathrm{~cm}^{-1}$ correlated to $\mathrm{C}=\mathrm{O}$ bond decreased in intensity and became wider. Formation of hydrogen bonding between phenolic hydroxyl group of curcumin and carboxylic hydroxy group and the carbonyl group in amide bond of chitosan could be the reason of the wider shape and decrease in intensity of Amide I bond. In fact, formation of hydrogen bonding increases the $\mathrm{C}-\mathrm{N}$ bond character and decreases the $\mathrm{C}=\mathrm{O}$ double bond character [46]. Also, the peak at $3496 \mathrm{~cm}^{-1}$ becomes wider and had a significant shift toward lower wave number at $3387 \mathrm{~cm}^{-1}$ indicating formation of strong intramolecular or intermolecular hydrogen bonding. 


\subsection{Entrapment efficiency}

Positive amino groups of chitosan can easily form polyelectrolyte complexes with negatively charged phenolic groups of curcumin by means of electrostatic interactions; therefore chitosan has the potential to entrap a high amount of curcumin. The entrapment efficiency of curcumin in chitosan nanodroplets was $49.1 \%$ (formulation Cur-NDs-1). The positive zeta potential value of drug-loaded nanodroplets indicated that all the sites with positive charge on the chitosan shell are not linked with curcumin molecules; therefore chitosan molecules still had the potential to entrap curcumin.

The entrapment efficiency increased by addition of $0.15 \mathrm{v} / \mathrm{v} \%$ Tween 20 to the emulsion system (from $45.1 \%$ to $77.8 \%$ ) but further increase in Tween 20 concentration resulted in the decreased entrapment efficiency $(61.3 \%$ ) (Table 1). The similar result was observed in our previous work [43].

\subsection{Stability of nanodroplets}

The co-surfactant nanodroplets showed good stability over three months at 4 and $25^{\circ} \mathrm{C}$. Nearly no changes in size and entrapment efficiency were observed for nanodrplets with co-surfactant (formulation Cur-NDs-2) (Fig. 3), while nanodroplets without co-surfactant inclusion (lecithin only) showed stability loss by coalescence as temperatures increased (formulation Cur-NDs-1). Lecithin degradation seems to be responsible for the aging of PFH droplets.

The surfactant influence is usually depended on the surfactant structure and the hydrophiliclipophilic balance (HLB). The hydrophile-lipophile balance (HLB) is commonly used to indicate the emulsifying property of surfactants. Theoretically, surfactants with higher HLB values have better solubility in water, and surfactants with HLB values higher than 7 are usually used to form oil-in-water emulsions [47]. Usually, coalescence of emulsion droplets decreases as HLB increases. Nonionic surfactants stabilize oil-in-water emulsions through short-range steric forces, which prevent droplets from getting close enough to aggregate. At higher HLB values, the number of hydrophilic head groups that protruded into the aqueous phase and prevent the droplets coalescence, increases which results in higher stabilizing effect of surfactant $[48,49]$.

The higher stability of PFH nanodroplets prepared with inclusion of Tween 20 as co-surfactant could be attributed to higher HLB value of Tween 20 (16.7) compared to lecithin (8.0) [48].

\subsection{Stability of curcumin loaded into chitosan nanodroplets}

Stability of curcumin is very low at physiological $\mathrm{pH}$ due to its rapid hydrolytic degradation and biotransformation. Therefore, stability of entrapped curcumin was assessed to evaluate the effect curcumin entrapment into chitosan nanodroplets on its stability.

Fig. 4 shows the stability of curcumin loaded in chitosan nanodroplets in phosphate-buffered solution at $\mathrm{pH}$ of 7.4 (formulation Cur-NDs-2). Curcumin in its nanoformulation form had a 
relatively good stability (90\%), while free curcumin showed rapid stability lost within $24 \mathrm{~h}$, and over $90 \%$ of curcumin molecules degraded during this time. These results indicated that the stability of curcumin could be increased by entrapment into a protective carrier like chitosan nanodroplets.

\subsection{Nanodroplets as ultrasound-responsive drug delivery agents}

The in vitro release profile (Table 1) of curcumin from chitosan nanodroplets (formulation CurNDs-2) at $\mathrm{pH}, 7.4$ indicated that curcumin was tightly retained by the nanodroplets and only $21.4 \%$ of curcumin released after $24 \mathrm{~h}$ at $\mathrm{pH} 7.4$ while in an acidic environment with $\mathrm{pH} 5.0(\mathrm{pH}$ of endosomal/cytosomal environment), the cumulative amount of curcumin release increased and about $46.2 \%$ of drug released from nanodroplets. According to these results, the release of curcumin from nanodroplets at normal $\mathrm{pH}$ was very low which is desired in controlled drug delivery to eliminate the side effects of therapeutic agent.

Ultrasound-aided drug release was also investigated. When the nanodroplets were incubated at $37{ }^{\circ} \mathrm{C}$ without ultrasound exposure, no curcumin released from the nanodroplets during $10 \mathrm{~min}$, thus indicating strong drug retention by the nanodroplets shell. Ultrasound exposure triggered the release of $63.5 \%$ curcumin from formulation Cur-NDs-2 within 4 min (Fig. 5) indicating acoustic droplet vaporization of nanodroplets. The rate of curcumin release was higher in the first minutes of sonication, and by further ultrasound exposure, drug release continued but with slower trend. This demonstrates the efficient ADV of nanodroplets at the beginning of sonication. Also, increase in curcumin release over time indicated that curcumin release was a function of the degree of ADV.

Besides, the higher drug release of formulation Cur-NDs-2 with higher PFH content compared to Cur-NDs-4 showed that ultrasound active drug release was directly proportional to the content of PFH. Since PFH serves as the template core in nanodroplet emulsion, increasing the amount of PFH results in formation of nanodroplets with bigger PFH core and thinner polymeric shell [31], which can have stronger droplet expansion and eventually more efficient drug release under the action of ultrasound. The schematic of ADV and ultrasound active drug release can be seen in $[7]$.

\subsection{Nanodroplets as ultrasound contrast agents}

The potential of chitosan/PFH nanodroplets to convert into microbubble under the action of ultrasound for contrast-enhanced imaging was investigated. B-mode images showed that (Fig. $6 \mathrm{a}, \mathrm{b}, \mathrm{c})$ the echogenicity of vaporized nanodroplets at the frequency of $1 \mathrm{MHz}$ was directly proportional to the droplet concentration. The signal to noise ratio (SNR) value decreased from $40.1 \mathrm{~dB}$ to $12.6 \mathrm{~dB}$ when the nanodroplet concentration reduced from $10^{5}$ to $10^{3}$ (Fig. $6 \mathrm{~d}$ ).

Acoustic droplet vaporization results in formation of large microbubble (Fig. 6a,b,c) with significantly higher acoustic impedance compared to small nanodroplets which are highly 
echogenic and generate high ultrasound contrast. These results demonstrated that curcuminloaded chitosan/PFH nanodroplets can be detected even at low concentrations and serve as highly sensitive theranostic agents for simultaneous tumor imaging and therapy.

\subsection{Hemolytic activity}

In vitro hemolysis test was performed to evaluate the toxicity of curcumin-loaded nanodroplets before in-vivo experiments. Fig. 7a shows the percentages of hemolysis activity of free curcumin, curcumin-loaded and empty nanodroplets. No hemolytic activity was observed for empty nanodroplets and hemolytic activity of curcumin-loaded nanodroplets was negligible compared to free curcumin.

Accumulation of drug molecule into the RBC increases the osmotic pressure and absorption of water into the cell, which results in the breakdown of cell membrane [50]. The small molecules of free curcumin can easily penetrate into RBC and cause cell death, while the bigger size of curcumin-loaded nanodroplets eliminates their chance to enter the RBC and subsequently decreases their hemolysis activity. In addition, the nanodroplets are covered by a chitosan shell which is a biocompatible non-toxic natural polymer. Therefore, curcumin-loaded nanodroplets compared to free curcumin, are more hemocompatible.

\subsection{In-vitro cytotoxicity of ADV-targeted nanodroplets}

The cytotoxic effect of curcumin-loaded nanodroplets with and without sonication was examined by MTT assay on 4T1 human breast cancer cells. Cytotoxicity showed dose-dependency. The cytotoxicity of all samples increased by increase in curcumin concentration (Fig. 7b).

Sonication significantly increased cytotoxicity of curcumin-loaded nanodroplets $\left(\mathrm{p}^{*}<0.05\right)$. Sonication of curcumin-loaded droplets resulted in $92.1 \%$ cytotoxicity while the cytotoxicity of nanodroplets without sonication was about 55.5\% at $.4 \mu \mathrm{g} / \mathrm{ml}$ (Fig. $7 \mathrm{~b}$ ) after $24 \mathrm{~h}$ of incubation. This significant change in cytotoxicity of nanodroplets indicated the effective curcumin release due to ADV. Acoustic droplet vaporization and oscillation and collapse of microbubbles resulted in triggered drug release from the microbubbles and enhanced permeability of cell membranes via strong and transient mechanical force which eventually enhanced intracellular drug uptake [51]. These result also indicated that before sonication, the drug was tightly retained by the nanodroplets and that was the reason of lower cytotoxicity of nanodroplets.

No cytotoxicity was observed for the 4T1 cells incubated with blank nanodroplets at the same amount used to deliver the highest concentration of curcumin $(1 \mathrm{mg} / \mathrm{ml})$ (data not shown).

The cytotoxicity of free curcumin dissolved in 5\% DMSO was also significantly lower than that of curcumin loaded into nanodroplets which could be because: (1) The significantly lower stability of curcumin in its free form, (2) and subsequently the lower cellular uptake of free curcumin. (3) The lipophilic nature of curcumin which results in easily incorporation of 
curcumin into the cell membrane [52]. This eliminates the diffusion of curcumin through the membrane, while curcumin-loaded nanodroplets enter the cell via endocytosis.

The results demonstrated that ultrasound-responsive chitosan nanodroplets not only are safe drug carriers but also improve the anti-cancer effects of curcumin, due to enhancing the stability of curcumin and their ability to release entrapped curcumin in a controlled and triggered manner which results in the effective killing of cancer cells.

\section{Conclusion}

Stable ultrasound-responsive chitosan-shelled perfluorohexane nanodroplets were successfully prepared for imaged-guided controlled curcumin delivery. The nanodroplets efficiently entrapped curcumin molecules and released them in control manner under the action of ultrasound which was correlated to the concentration of perflourohexane and degree of acoustic droplet vaporization. The nanodroplets showed strong ultrasound contrast even at low concentration. Cytotoxicity study on 4T1 human breast cancer cells indicated the significantly enhanced anti-tumor activity of curcumin-loaded nanodroplets under the action of $1 \mathrm{MHz}$ ultrasound. Also drug-loaded nanodroplets showed no hemolytic activity. Therefore, it could be concluded that ultrasound responsive chitosan/perfluorohexane nanodroplets are promising nanocarriers for theranostic applications.

\section{Acknowledgement}

The project described was supported by Iranian National Science Foundation.

\section{References}

[1] S. Mitragotri, Healing sound: the use of ultrasound in drug delivery and other therapeutic applications, Nat. Rev. Drug Discov. 4 (2005) 255-260.

[2] D.L. Miller, M.A. Averkiou, A.A. Brayman, E.C. Everbach, C.K. Holland, J.H. Wible Jr., J. Wu, Bioeffects considerations for diagnostic ultrasound contrast agents, J. Ultrasound Med. 27 (2008) 611-6328 quiz 633-616.

[3] S. Qin, C.F. Caskey, K.W. Ferrara, Ultrasound contrast microbubbles in imaging and therapy: physical principles and engineering, Phys. Med. Biol. 54 (2009) R27-R57.

[4] K. Hynynen, Ultrasound for drug and gene delivery to the brain, Adv. Drug Deliv. Rev. 60 (2008) 1209-1217.

[5] O.D. Kripfgans, J.B. Fowlkes, D.L. Miller, O.P. Eldevik, P.L. Carson, Acoustic droplet vaporization for therapeutic and diagnostic applications, Ultrasound Med Biol 26 (2000) 1177-1189.

[6] K.I. Kawabata, N. Sugita, H. Yoshikawa, T. Azuma, S.I. Umemura, Nanoparticles with multiple perfluorocarbons for controllable ultrasonically induced phase shifting, Jpn J Appl Phys 44 (2005) 4548-4552. 
[7] N.Y. Rapoport, A.M. Kennedy, J.E. Shea, C.L. Scaife, K.H. Nam, Controlled and targeted tumor chemotherapy by ultrasound-activated nanoemulsions/microbubbles. J Control Release 138 (2009) 268-276.

[8] M.L. Fabiilli, K.J. Haworth, I.E. Sebastian, O.D. Kripfgans, P.L. Carson, J.B. Fowlkes, Delivery of chlorambucil using an acoustically-triggered perfluoropentane emulsion, Ultrasound Med Biol 36 (2010) 1364-1375.

[9] K.W. Ferrara, Driving delivery vehicles with ultrasound, Adv Drug Deliv Rev 60 (2008) 1097-1102.

[10] P. Prentice, A. Cuschieri, K. Dholakia, M. Prausnitz, P. Campbell, Membrane disruption by optically controlled microbubble cavitation, Nat Phys 1 (2005) 107-110.

[11] M. Zhang, M.L. Fabiilli, K.J. Haworth, J.B. Fowlkes, O.D. Kripfgans, W.W. Roberts, et al, Initial investigation of acoustic droplet vaporization for occlusion in canine kidney, Ultrasound Med Biol 36 (2010) 1691-1703.

[12] J. Huang, J.S. Xu, R.X. Xu, Heat-sensitive microbubbles for intraoperative assessment of cancer ablation margins, Biomaterials 31 (2010) 1278-1286.

[13] P. Anand, A.B. Kunnumakkara, R.A. Newman, B.B. Aggarwal, Bioavailability of curcumin: problems and promises, Molecular Pharmaceutics 4 (2007) 807-818.

[14] P. Anand, C. Sundaram, S. Jhurani, A.B. Kunnumakkara, B.B. Aggarwal, Curcumin and cancer: an "old age" disease with an "age-old” solution, Cancer Letters 267 (2008) 133-164.

[15] A.J. Ruby, G. Kuttan, K.D. Babu, K.N. Rajasekharan, R. Kuttan, Anti-tumour and antioxidant activity of natural curcuminoids, Cancer Letters 94 (1995) 79-83.

[16] J.S. Wright, Predicting the antioxidant activity of curcumin and curcuminoids, Journal of Molecular Structure: THEOCHEM 591 (2002) 207-217.

[17] R.C. Srimal, B.N. Dhawan, Pharmacology of diferuloyl methane (curcumin), a non-steroidal anti-inflammatory agent, Journal of Pharmacy and Pharmacology 25 (1973) 447-452.

[18] R.K. Maheshwari, A.K. Singh, J. Gaddipati, R.C. Srimal, Multiple biological activities of curcumin: a short review, Life Sciences 78 (2006) 2081-2087.

[19] P.K. Shukla, V.K. Khanna, M.M. Ali, M.Y. Khan, R.C. Srimal, Anti-ischemic effect of curcumin in rat brain, Neurochemical Research 33 (2008) 1036-1043.

[20] S. Y. Park, Potential therapeutic agents against Alzheimer's disease from natural sources, Archives of Pharmacal Research 33 (2010) 1589-1609.

[21] T. Dorai, Y.C. Cao, B. Dorai, R. Buttyan, A.E. Katz, Therapeutic potential of curcumin in human prostate cancer. III. Curcumin inhibits proliferation, induces apoptosis, and inhibits angiogenesis of LNCaP prostate cancer cells in vivo, The prostate 47 (2001) 293-303.

[22] W. Chearwae, S. Anuchapreeda, K. Nandigama, S.V. Ambudkar, P. Limtrakul, Biochemical mechanism of modulation of human P-glycoprotein (ABCB1) by curcumin I, II, and III purified from Turmeric powder. Biochemical Pharmacology 68 (2004) 2043-2052.

[23] Y. Kaminaga, A. Nagatsu, T. Akiyama, N. Sugimoto, T. Yamazaki, T. Maitani, H. Mizukami, Production of unnatural glucosides of curcumin with drastically enhanced water solubility by cell suspension cultures of Catharanthus roseus, FEBS Letters 555 (2003) 311-316.

[24] V. Ravindranath, N. Chandrasekhara, Absorption and tissue distribution of curcumin in rats, Toxicology 16 (1980) 259-265. 
[25] N. Rapoport, Physical stimuli-responsive polymeric micelles for anti-cancer drug delivery, Prog. Polym. Sci. 32 (2007) 962-990.

[26] N. Rapoport, Z. Gao, A. Kennedy, Multifunctional nanoparticles for combining ultrasonic tumor imaging and targeted chemotherapy, J. Natl Cancer Inst. 99 (2007) 1095-1106.

[27] N. Rapoport, D.A. Christensen, A.M. Kennedy, K.H. Nam, Cavitation properties of block copolymer stabilized phase-shift nanoemulsions used as drug carriers, Ultrasound Med. Biol. 36 (2010) 419-429.

[28] N. Rapoport, A.M. Kennedy, J.E. Shea, C.L. Scaife, K.H. Nam, Ultrasonic nanotherapy of pancreatic cancer: lessons from ultrasound imaging, Mol. Pharm. 7 (2010) 22-31.

[29] N.Y. Rapoport, A.L. Efros, D.A. Christensen, A.M. Kennedy, K.H. Nam, Microbubble generation in phaseshift nanoemulsions used as anticancer drug carriers, Bub Sci. Eng. Technol. 1 (2009) 31-39.

[30] K.H. Nam, D.A. Christensen, A.M. Kennedy, N. Rapoport, Acoustic droplet vaporization, cavitation, and therapeutic properties of copolymer-stabilized perfluorocarbon nanoemulsions, Am. Inst. Phys. Conf. Proc. 1113 (2009) 124-128.

[31] Z. Gao, A.M. Kennedy, D.A. Christensen, N. Rapoport, Drug-loaded nano/ microbubbles for combining ultrasonography and targeted chemotheraphy, Ultrasonics 48 (2008) 260-270.

[32] C.H. Wang, S.T. Kang, Y.H. Lee, Y.L. Luo, Y.F. Huang, C.K. Yeh, Aptamer-conjugated and drug-loaded acoustic droplets for ultrasound theranosis, Biomaterials 33 (2012) 1939-1947

[33] S.R. Sirsi, C. Fung, S. Garg, M.Y. Tianning, P.A. Mountford, M.A. Borden, Lung surfactant microbubbles increase lipophilic drug payload for ultrasound-targeted delivery, Theranostics. 3 (2013) 409.

[34] M.L. Fabiilli, K.J. Haworth, N.H. Fakhri, O.D. Kripfgans, P.L. Carson, J.B. Fowlkes, The role of inertial cavitation in acoustic droplet vaporization, IEEE Trans. Ultrason. Ferr. 56 (5) (2009) 1006-1017.

[35] N. Reznik, M. Seo, R. Williams, E. Bolewska-Pedyczak, M. Lee, N. Matsuura, J. Gariepy, F.S. Foster, P.N. Burns, Optical studies of vaporization and stability of fluorescently labelled perfluorocarbon droplets, Phys. Med. Biol. 57 (21) (2012) 7205-7217.

[36] R. Singh, G.A. Husseini, W.G. Pitt, Phase transitions of nanoemulsions using ultrasound: Experimental observations, Ultrasonics Sonochemistry 19 (2012) 1120-1125.

[37] Y. Jia, Y. Hu, Y. Zhu, L. Che, Q. Shen, J. Zhang, X. Li, Oligoamines conjugated chitosan derivatives: synthesis, characterization, in vitro and in vivo biocompatibility evaluations, Carbohydrate Polymers 83 (2011) 1153-1161.

[38] O. Hiraku, Y. Machida, Biodegradation and distribution of water-soluble chitosan in mice, Biomaterials 20 (1999) 175-182.

[39] M.N.V. Ravi Kumar, A review of chitin and chitosan applications, Reactive and Functional Polymers 46 (2000) 1-27.

[40] H. Yuan, L.J. Lu, Y.Z. Du, F.Q. Hu, Stearic acid-g-chitosan polymeric micelle for oral drug delivery: in vitro transport and in vivo absorption, Molecular Pharmaceutics 8 (2011) 225-238. 
[41] S.P. Mitra, Stabilizing effect of chitosan on curcumin from the damaging action of alkaline $\mathrm{pH}$ and ultraviolet light, Journal of Surface Science and Technology 24 (2008) 39-55.

[42] I. Pepic, J. Filipovic-Grcic, I. Jalsenjak, Bulk properties of nonionic surfactant and chitosan mixtures, Colloids and Surfaces A 336 (2009) 135-141.

[43] F. Baghbani, F. Moztarzadeh, J. Aghazadeh Mohandesi, F. Yazdian, M. Mokhtari Dizagi, S. Hamedi, Formulation design, preparation and characterization ofmultifunctional alginate stabilized nanodroplets, Int.1 J. of Biol. Macromolec. 89 (2016) 550-558.

[44] G.D. Rosa, R. Iommelli, M.I. La Rotonda, et al., Influence of the co-encapsulation of different non-ionic surfactants on the properties of PLGA insulin-loaded microspheres, J Control Release 69 (2000) 283-295.

[45] L. Du, Y. Jin, W. Zhou, J. Zhao, Ultrasound-triggered drug release and enhanced anticancer effect of doxorubicin-loaded poly(D,L-lactide-co-glycolide)-methoxy-poly (ethylene glycol) nanodroplets. Ultrasound in Medicine \& Biology 37 (2011) 1252-1258.

[46] J. Xiao, S. Nian, Q. Huang, Assembly of kafirin/carboxymethyl chitosan nanoparticles to enhance the cellular uptake of curcumin, FOOD HYDROCOLLOID 51 (2015) 166-175.

[47] P. Walstra, Surface phenomena. In Physical Chemistry of Foods; CRC Press: New York, Basel, (2002) p 348.

[48] Surfactant 172, available at http://class.fst.ohio-state.edu/fst621/lectures/emuls.htm.

[49] Sorbitan Fatty Acid Ester, available at http://www.surfactant.co.kr/surfactants/sorbitan.html.

[50] D. Lu, Jie L.Y. Fan, Z. Gu, X. Zhang, In Vivo Evaluation of a pH-Sensitive Pullulan-Doxorubicin Conjugate. Adv. Eng. Mater.12(9) (2010) 496-503.

[51] N. Rapoport, Phase-shift, stimuli-responsive perfluorocarbon nanodroplets for drug delivery to cancer Wiley Interdiscip Rev Nanomed Nanobiotechnol. 4(5) (2012) 492-510.

[52] R. Baskaran, T. Madheswaran, P. Sundaramoorthy, H. M. Kim, B.K. Yoo, Entrapment of curcumin into monoolein-based liquid crystalline nanoparticle dispersion for enhancement of stability and anticancer activity, International Journal of Nanomedicine 9 (2014) 3119-3130. 
Table legend:

Table 1. Particle size, zeta potential, entrapment efficiency, and cumulative amount of drug released from curcumin-loaded chitosan/PFH nanodroplets.

Figure captions:

Fig 1. Schematic of ultrasound set up used for ultrasound-aided drug release and cytotoxicity experiment.

Fig. 2. (a) Morphology of curcumin-loaded chitosan shelled PFH nanodroplets (TEM image), (b) FTIR spectra of curcumin, chitosan and curcimin-loaded chitosan nanodroplets.

Fig 3. Nanodroplets Stability, changes in a) particle size and b) entrapment efficiency of the curcumin-loaded nanodroplets incubated at 4 and $37^{\circ} \mathrm{C}$ for predetermined time intervals.

Fig 4. Curcumin stability. Stability of free curcumin and curcumin nanodrplets in PBS (pH 7.2). Means $\pm \mathrm{SD}(\mathrm{n}=3)$.

Fig 5. In-vitro release profiles of curcumin from chitosan/PFH nanodroplets (formulations CurND-2 and Cur-ND-4) without ultrasound (control) and with ultrasound exposure (1 MHz, 2 $\left.\mathrm{W} / \mathrm{cm}^{2}\right)$ in $\mathrm{pH} 7.4$, at $37^{\circ} \mathrm{C}$ for 7 min, Means $\pm \mathrm{SD}(\mathrm{n}=3)$.

Fig 6. a,b,c) Increase in ADV of chitosan/PFH nanodroplets as a function of $\mathrm{PFH}$ volume ratio (B-mode ultrasound images), d) SNR profile of chitosan/PFH nanodroplets under the action of ultrasound as a function of $\mathrm{PFH}$ volume ratio.

Fig 7. (a) Hemolytic activities of blank nanodrplets, free curcumin and curcumin-loaded nanodroplets (formulation Cur-ND-2); Means $\pm \mathrm{SD}(\mathrm{n}=3)$; (b) Cytotoxicity of free curcumin, Cur-NDs and Cur-ND-US against 4T1 cells for $24 \mathrm{~h}$; Means $\pm \mathrm{SD}(\mathrm{n}=5)$. * $\mathrm{p}<0.05$; 
Table 1. Comparison of particle size, entrapment efficiency, and cumulative amount of drug released from curcumin-loaded $\mathrm{PFH} /$ chitosan nanodroplets prepared using various formulation variables.

\begin{tabular}{|c|c|c|c|c|c|c|c|c|c|c|}
\hline $\begin{array}{c}\text { Formulation } \\
\text { code }\end{array}$ & $\begin{array}{c}\text { Chitosan } \\
(\% \mathrm{w} / \mathrm{v})\end{array}$ & $\begin{array}{c}\text { Lecithin } \\
(\% \mathrm{w} / \mathrm{v})\end{array}$ & $\begin{array}{c}\text { Tween20 } \\
(\% \mathrm{v} / \mathrm{v})\end{array}$ & $\begin{array}{c}\text { Curcumin } \\
(\mu \mathrm{g})\end{array}$ & $\begin{array}{c}\mathrm{PFH} \\
(\% \mathrm{v} / \mathrm{v})\end{array}$ & $\begin{array}{c}\text { Particle } \\
\text { Size } \\
(\mathrm{nm} \pm \\
\mathrm{SD})\end{array}$ & $\begin{array}{c}\text { Mean } \\
\text { Polydispersity } \\
\text { index }\end{array}$ & $\begin{array}{c}\text { zeta- } \\
\text { potential }\end{array}$ & $\begin{array}{c}\text { Entrapment } \\
\text { Efficiency } \\
(\% \pm \mathrm{SD})\end{array}$ & $\begin{array}{c}\text { Cumulative } \\
\text { Released at } \\
24 \mathrm{H}(\% \pm \\
\mathrm{SD})\end{array}$ \\
\hline Blank-NDs & 0.2 & 0.1 & 0 & 0 & 8 & $163 \pm 4$ & $0.32 \pm 0.07$ & $+64.01 \pm 2.1$ & 0 & 0 \\
\hline Cur-NDs-1 & 0.2 & 0.1 & 0 & 400 & 8 & $255 \pm 7$ & $0.315 \pm 0.5$ & $+28.37 \pm 1.4$ & $49.1 \pm 1.4$ & - \\
\hline Cur-NDs-2 & 0.2 & 0.1 & 0.15 & 400 & 8 & $101.2 \pm 4$ & $0.352 \pm 0.01$ & $+26.13 \pm 1.6$ & $77.8 \pm 2.6$ & $21.4 \pm 1.7$ \\
\hline Cur-NDs-3 & 0.2 & 0.1 & 0.3 & 400 & 8 & $184.8 \pm 9$ & $0.315 \pm 0.08$ & $+27.71 \pm 0.7$ & $61.3 \pm 2.2$ & - \\
\hline Cur-NDs-4 & 0.2 & 0.1 & 0.15 & 400 & 3 & $68.7 \pm 11$ & $0.364 \pm 0.05$ & $+25.98 \pm 1.1$ & $71.1 \pm 3.0$ & - \\
\hline
\end{tabular}


a)

$4 \mathrm{~mm}$

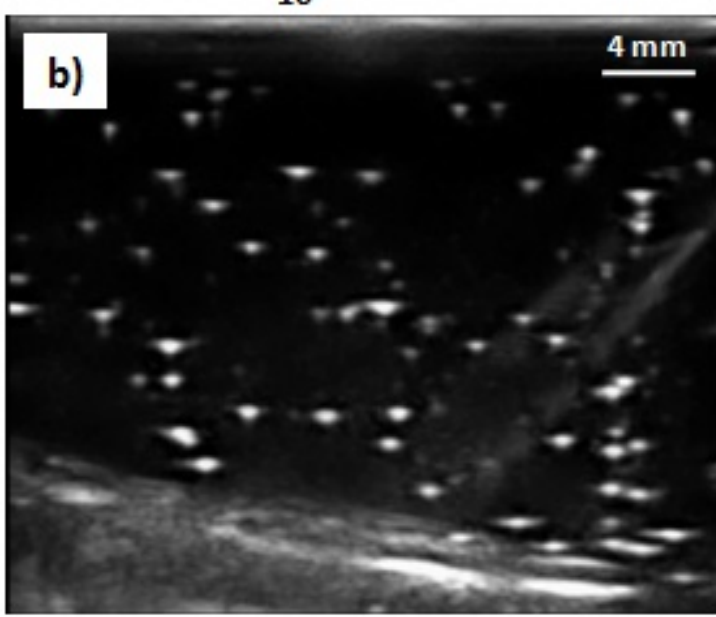

c)

$4 \mathrm{~mm}$ $\underline{4 \mathrm{~mm}}$

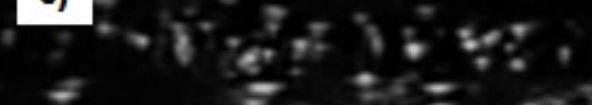

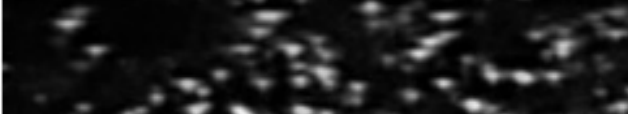

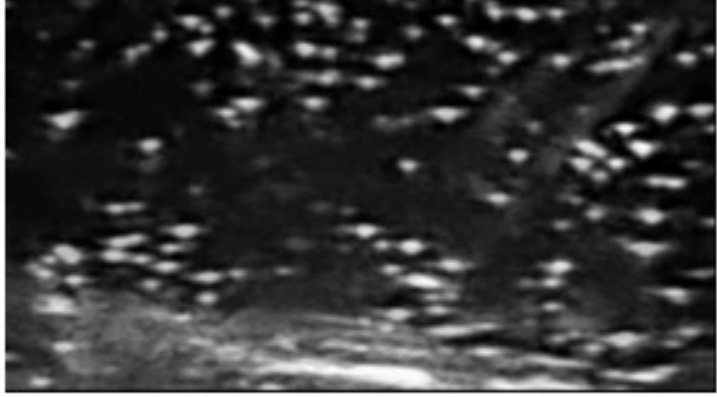
$10^{5}$
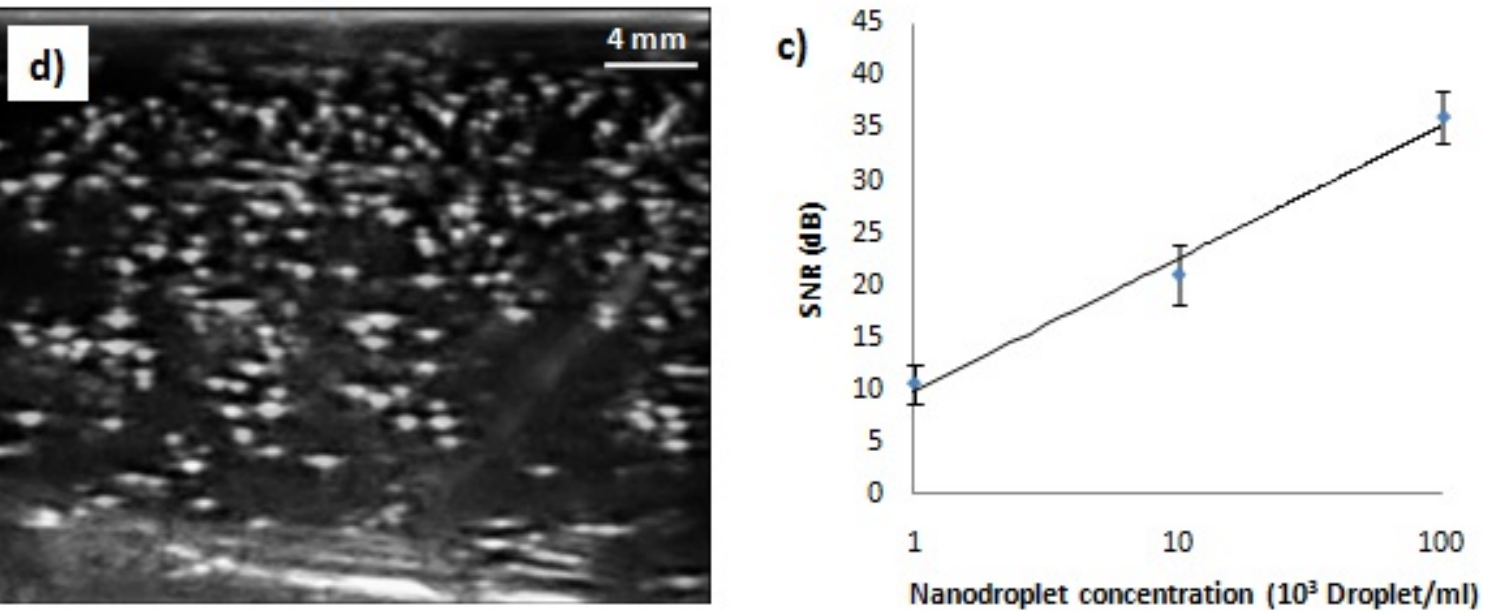


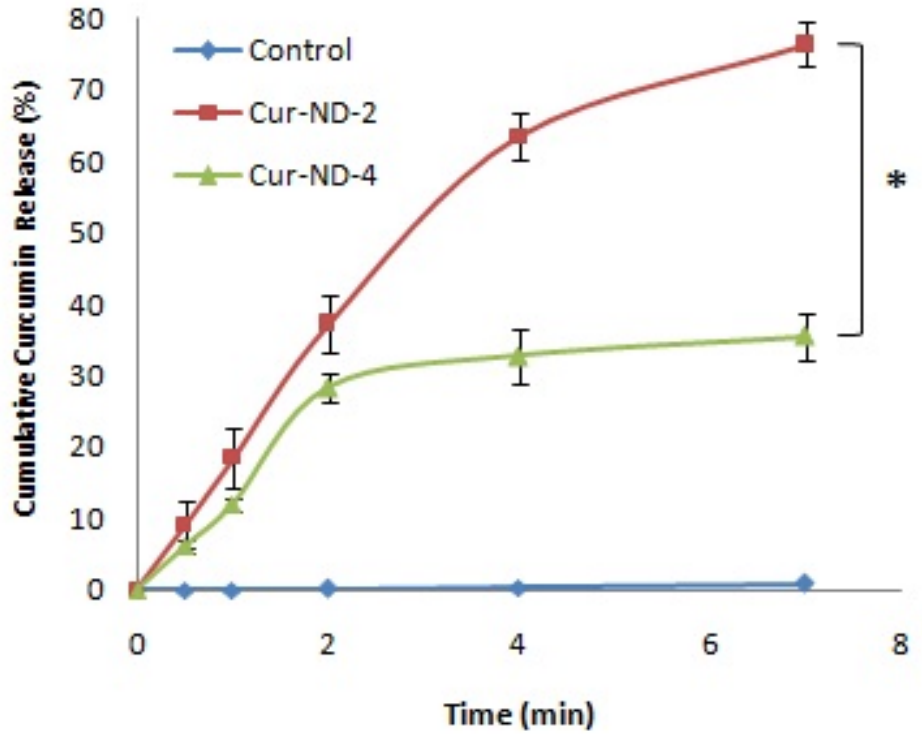




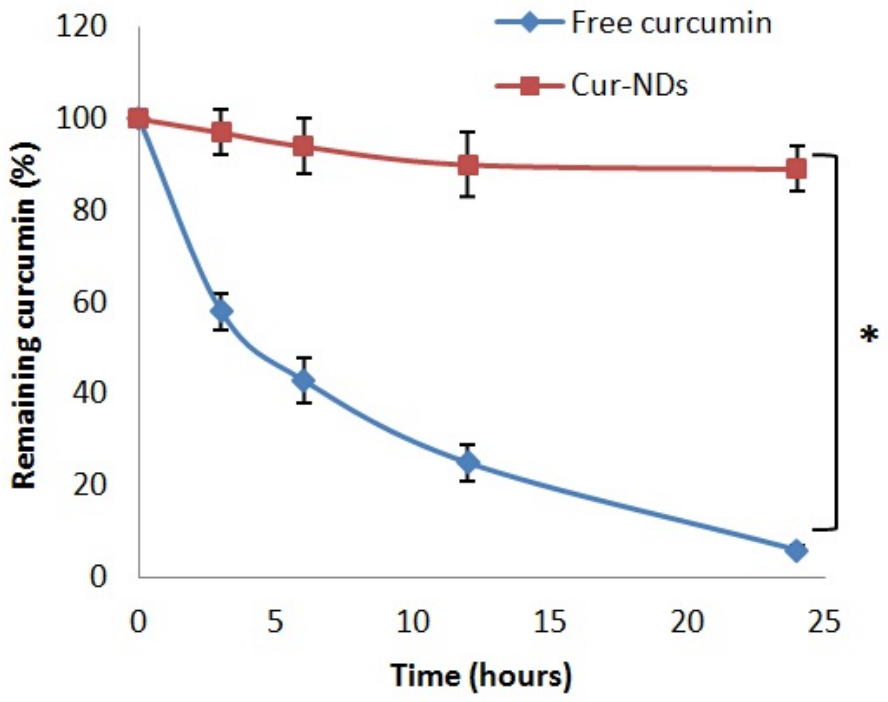


a) 450

400

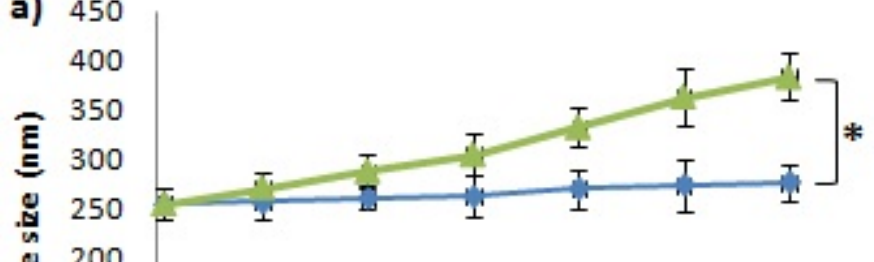

200

150

100

50

0

b)

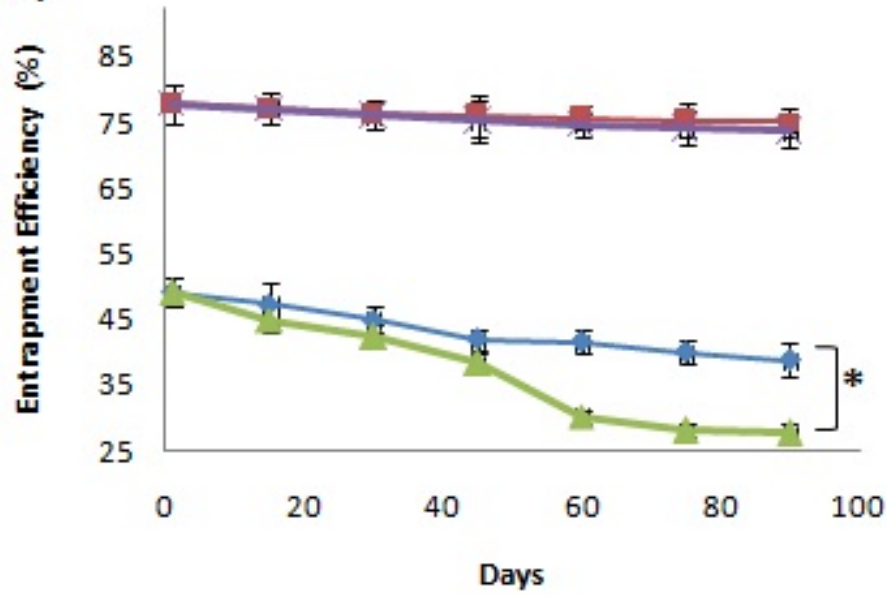

$\longrightarrow$ ND- $1,4^{\circ} \mathrm{C}$

$-\mathrm{ND}-2,4^{\circ} \mathrm{C}$

-ND-1, $25^{\circ} \mathrm{C}$

$\longrightarrow \mathrm{ND}-2,25^{\circ} \mathrm{C}$ 


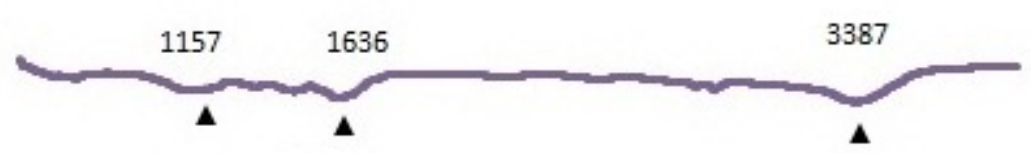

Cur-chitosan
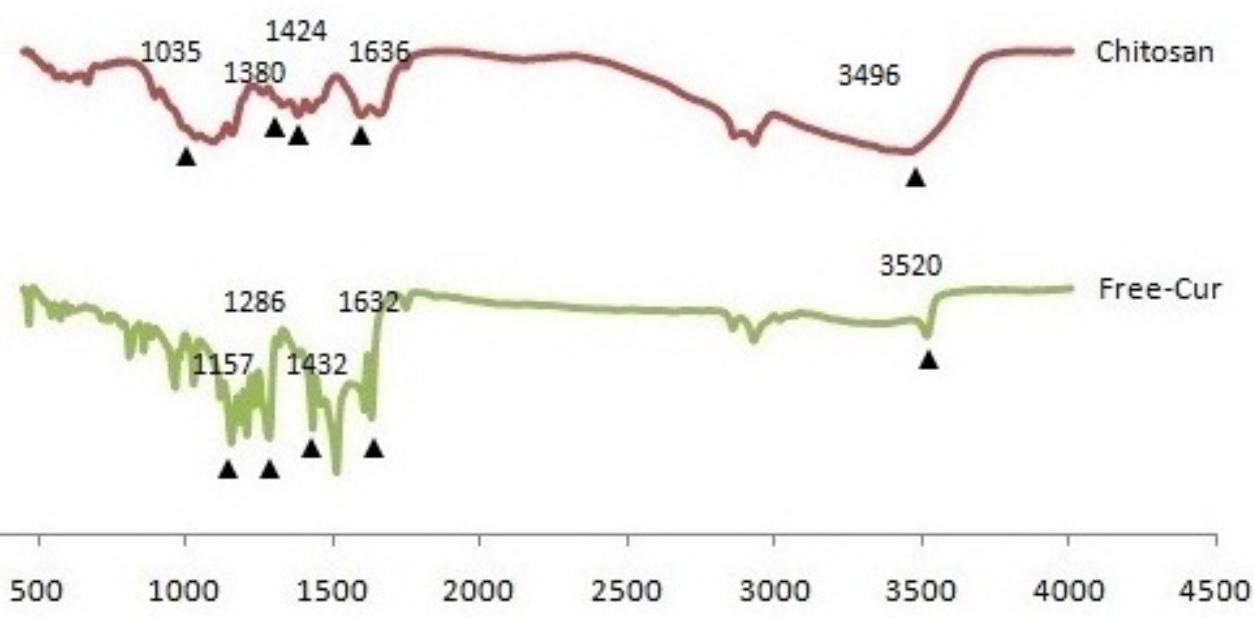

Wavenumber $\left(\mathrm{cm}^{-1}\right)$ 
Cell culture Plate

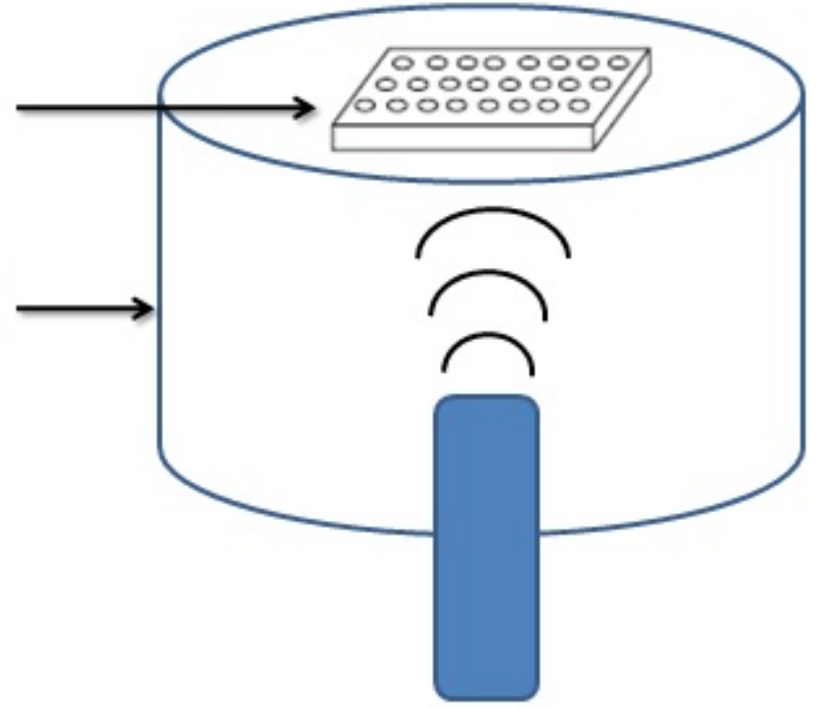

Ultrasound Probe 